\title{
Author Correction: Evaluation of deep learning in non-coding RNA classification
}

Noorul Amin (1), Annette McGrath and Yi-Ping Phoebe Chen (1)

Correction to: Nature Machine Intelligence https://doi.org/10.1038/s42256-019-0051-2, published online 13 May 2019.

In the version of this Analysis originally published, the authors used a non-default setting for one of the software packages they compare. Because of this they have made the following changes to the text.

In the subsection 'Evaluation results', the text 'IncADeep shows the lowest performance in all the metrics, but has the highest recall of 0.988 ' has been changed to 'The full-length model of lncADeep showed the lowest performance, except for a high recall of 0.988 . However, our later analysis revealed that the partial-length model of lncADeep performs better than lncRNAnet and lncFinder in 5 out of 6 metrics'.

In the same subsection, the text 'The lncADeep PR curve is just above the no-skill model, with an average precision of 0.52 and 0.56 on lncGH and lncGM' has been changed to 'The average precision of the full-length lncADeep is 0.52 and 0.56 on lncGH and lncGM'.

The section 'Conclusions', the text 'IncADeep yielded the best recall but showed poor overall performance' has been changed to 'The full-length model of lncADeep yielded the best recall but for other metrics, the performance was not optimal. The performance of the partial-length model of lncADeep was found to be significantly better and details can be found in the correspondence to this Analysis'.

Published online: 13 January 2020

https://doi.org/10.1038/s42256-019-0143-z

๑) Springer Nature Limited 2020 